\title{
Highly ordered silicide ripple patterns induced by medium-energy ion irradiation
}

\author{
A. Redondo-Cubero $\odot,{ }^{1}$ F. J. Palomares $\odot,{ }^{2}$ R. Hübner $\odot,{ }^{3}$ R. Gago $\odot,{ }^{2}$ and L. Vázquez $\odot^{2, *}$ \\ ${ }^{1}$ Electronics and Semiconductors Group, Departamento de Física Aplicada, Universidad Autónoma de Madrid, 28049 Madrid, Spain \\ ${ }^{2}$ Instituto de Ciencia de Materiales de Madrid, Consejo Superior de Investigaciones Científicas, E-28049 Madrid, Spain \\ ${ }^{3}$ Helmholtz-Zentrum Dresden-Rossendorf, Institute of Ion Beam Physics and Materials Research, D-01328 Dresden, Germany
}

(Received 20 May 2020; revised 14 July 2020; accepted 14 July 2020; published 11 August 2020)

\begin{abstract}
We study the nanopatterning of silicon surfaces under near-normal 40-keV $\mathrm{Ar}^{+}$sputtering with simultaneous Fe oblique codeposition. The ion-beam incidence was kept at $15^{\circ}$, for which no pattern is produced in the absence of metal incorporation. Morphological and compositional analyses were performed by atomic force microscopy, in its morphological and electrical modes, Rutherford backscattering spectrometry, x-ray photoelectron spectroscopy, scanning Auger, as well as transmission and scanning electron microscopy. Initially, nanodot structures randomly emerge, which, with increasing ion fluence, become progressively aligned along the perpendicular direction to the Fe flux. With increasing fluence, they coalesce, leading to a ripple pattern. The pattern dynamics and characteristics are faster and enhanced, respectively, as the distance to the metal source decreases (i.e., as the metal content increases). For the highest metal flux, the ripples can become rather large (up to $18 \mu \mathrm{m}$ ) and straighter, with few defects, and a pattern wavelength close to $500 \mathrm{~nm}$, while keeping the surface roughness close to $15 \mathrm{~nm}$. Furthermore, for a fixed ion fluence, the pattern order is improved for higher metal flux. In contrast, the pattern order enhancement rate with ion fluence does not depend on the metal flux. Our experimental observations agree with the predictions and assumptions of the model by Bradley [R. M. Bradley, Phys. Rev. B 87, 205408 (2013)] Several compositional and morphological studies reveal that the ripple pattern is also a compositional one, in which the ripple peaks have a higher iron silicide content, in agreement with the model. Likewise, the ripple structures develop along the perpendicular direction to the Fe flux, and the pattern wavelength increases as the metal flux decreases with a behavior qualitatively consistent with the model predictions.
\end{abstract}

DOI: 10.1103/PhysRevB.102.075423

\section{INTRODUCTION}

Ion-beam sputtering (IBS) has proven to be a powerful technique to produce nanopatterns on different surfaces in a short time and over relatively large areas [1]. In the last years, considerable advances have been made in the understanding of the fundamental mechanisms involved in IBS pattern formation and development [2-11]. From the experimental point of view, two main branches can be clearly distinguished: IBS patterning without and with simultaneous supply of foreign species (mainly metals) [2]. In the first mode, referred to as "under clean conditions", usually ripple morphologies are induced on the surface for incidence angles of the ion beam with respect to the surface normal $(\theta)$ above a threshold value (usually close to $45^{\circ}-50^{\circ}$ ) $[2,6,7,12]$. In this mode, the IBS patterning of metal surfaces is a particular case since ripple orientation depends on the bombardment geometry and temperature $[13,14]$. In the second mode, a larger variety of pattern morphologies can be induced such as dots or ripples [15-21]. Initially, the first reports concerned the production of dot patterns using Mo as seeding metal [22,23]. Silicon is the main target surface used for IBS patterning under both experimental conditions [24]. In particular, for those experiments with simultaneous metal supply, one of the most

*Corresponding author: 1vb@icmm.csic.es employed configurations consists of a target surface (usually silicon) with a metal plate placed at one of its ends that is the source of metal codeposition. The incoming ion beam hits both the target surface and the metal plate. This setup causes that, while the target surface is being eroded by the ion beam, the metal atoms sputtered, predominantly perpendicular from the plate by the same ion beam, eventually land on the target surface. As stated in previous works [18-20,25-30], we can assume that the larger the distance from the plate, the lower the Fe flux. This fact triggers new processes, which are absent in IBS under clean conditions, and are the source of the great variety of patterns that can be induced. Specifically, it has been proposed that a key process behind patterning of silicon surfaces by IBS with metal codeposition is the formation of silicides on the surface [24,26,28,31-33]. However, there are other works where the key role of silicide formation is questioned [34,35].

Most of the experimental studies performed on this second mode have been carried out using low-energy ion beams (with energies equal to or below $5 \mathrm{keV}$ ). Some of the present authors reported the first study on this configuration using mediumenergy ion beams $\left(40 \mathrm{keV} \mathrm{Ar}^{+}\right)$[27]. In principle, the employment of medium-energy ions will imply a larger ion penetration length, ion-induced amorphization, and sputtering rates that could lead to different pattern formation and dynamics. Here, we extend this former work by changing the incidence angle of the ion beam on the target from $60^{\circ}$ [27] to $15^{\circ}$. The 
physical motivation behind this choice is that, at $\theta=60^{\circ}$ the ion beam does produce a ripple pattern on the silicon surface without the need of simultaneous metal supply [36], i.e., it is above the threshold angle for pattern formation $[2,6,7]$. However, $\theta=15^{\circ}$ lies well below this threshold and, therefore, the silicon surface remains featureless under bombardment at this angle under clean conditions. As a consequence, the physical mechanisms for triggering the pattern formation are dominated by the concurrent metal incorporation in terms of deposition rate, directionality, lateral distribution and, eventually, chemical reaction with the target atoms.

This change on the experimental conditions has important consequences because, as shown below, they are compatible with those considered in one of the main models addressing IBS patterning with impurity codeposition [37]. This fact allows us to compare the experimental data with those predicted and assumed in the model. Moreover, the better ordered patterns produced now, with respect to those reported earlier [27], make possible to provide contrasted data on the pattern ordering as a function of the IBS conditions. Thus, although we use a similar methodology [27], i.e., by analyzing the different morphologies as the ion fluence $(\Phi)$ increases at several distances from the metal source (i.e., for different Fe fluxes), the experimental conditions are such that allow us to address issues as important as the pattern order and the role of the metal flux, that may contribute to further the understanding of this kind of IBS patterning.

\section{EXPERIMENT}

\section{A. Sample production}

The irradiation experiments were performed with a $40-\mathrm{keV}$ $\mathrm{Ar}^{+}$beam extracted from a Danfysik 1090 ion implanter with a base pressure of $5 \times 10^{-6}$ mbar. The spot size is about $1.5 \times 1.5 \mathrm{~mm}^{2}$. The ions impinged on the single-crystal $\operatorname{Si}(100)$ targets $\left(1 \times 1 \mathrm{~cm}^{2}\right.$ in size $)$ at $\theta=15 \pm 5^{\circ}$ with respect to the surface normal with a current density of $34 \mu \mathrm{A} / \mathrm{cm}^{2}$ in the sample plane. Under these conditions, the average and maximum ion penetration ranges estimated by Stopping and range of ions in matter (SRIM) simulations are 46 and 100 $\mathrm{nm}$, respectively. A steel plate $(1.5 \mathrm{~mm}$ high) placed adjacent to the Si target acted simultaneously as Fe codeposition source and sample attachment to the holder. To obtain homogeneous irradiation, the focused beam was scanned with a magnetic $x-y$ sweeping system in such a way that both the Si surface and the steel target were bombarded. The irradiation times spanned from $20 \mathrm{~min}$ to $16 \mathrm{~h}$, implying $\Phi$ values up to $\sim 1.2 \times 10^{19}$ ions $/ \mathrm{cm}^{2}$.

\section{B. Sample characterization}

The resulting surface morphology after irradiation was imaged ex situ by atomic force microscopy (AFM) operating in the dynamic mode with Nanoscope IIIa equipment (Veeco) and with an Agilent PicoPlus 5500, the latter being able to operate in current-sensing mode (CS-AFM) [38]. Silicon cantilevers, with a nominal radius, $r$, of $8 \mathrm{~nm}$ and an opening angle smaller than $52^{\circ}$, were employed for the topographical measurements whereas Pt-coated tips (ANSCM-PT from APPNano) were used for the electrical modes. For CS-AFM imaging, a voltage bias with both polarities within the 3-9 $\mathrm{V}$ range was employed. These measurements were performed under nitrogen atmosphere to avoid contributions from the water vapor adsorbed on the surface. Likewise, Kelvin force microscopy (KFM) was carried out with the same cantilevers and under similar conditions. In the KFM measurements, the sample was grounded. KFM measures the contact potential difference, which is the difference between the work-function values of the imaged sample location and that of the tip.

The surface roughness, $\sigma$, was obtained from the AFM images using the equipment software. Other image analyses were made with the GWYDDION and IMAGEJ freeware packages [39-41]. In particular, the characteristic wavelength of the ripple pattern, $\lambda$, was obtained from the power spectral density (PSD) of the images as follows [24,42]. The PSD is defined as $\operatorname{PSD}(k, t)=\langle H(k, t) H(-k, t)\rangle$ where $H(k, t)$ is the Fourier transform of the surface height of the one-dimensional cut, $h(r, t)-\langle h\rangle$, where $\langle h\rangle$ stands for the average height of this profile, and $k$ is the spatial frequency. Since anisotropic ripple patterns run perpendicular to the ion-beam projection on the target surface, we have obtained the corresponding PSD curves along the projected direction of the ion beam $\left(\mathrm{PSD}_{\|}\right)$. Pattern formation is the result of the selection of a spatial frequency, $k_{0}$, and hence, a peak would appear in the $\mathrm{PSD}_{\|}$ curve. In this case, the pattern wavelength, $\lambda$, can be obtained from the relationship $k_{0}=1 / \lambda$.

Transmission electron microscopy (TEM) analysis in cross-sectional geometry was performed using an image- $\mathrm{C}_{\mathrm{s}^{-}}$ corrected Titan 80-300 microscope (FEI) operated at an accelerating voltage of $300 \mathrm{kV}$. Besides bright-field TEM imaging, element mapping using energy-filtered TEM based on the three-window-method at the Si- $L_{2,3}$, Ar- $L_{2,3}$, and Fe- $L_{2,3}$ edges was done employing a Gatan Imaging Filter 863. Prior to TEM analysis, the specimen mounted in a double-tilt lowbackground holder was placed for $10 \mathrm{~s}$ into a model 1020 Plasma Cleaner (Fischione) to remove possible contamination. Classical TEM cross sections of the sample glued together in face-to-face geometry using G2 epoxy glue (Gatan) were prepared by sawing (Wire Saw WS 22, IBS GmbH), grinding (MetaServ 250, Bühler), polishing (Minimet 1000, Bühler), dimpling (Dimple Grinder 656, Gatan), and final Ar ion milling (Precision Ion Polishing System PIPS 691, Gatan).

The Fe content along the nanopatterned surfaces for different ion fluences (irradiation times) was determined by means of Rutherford backscattering spectrometry (RBS). The probing beam was $2 \mathrm{MeV} \mathrm{He}^{+}\left(1 \mathrm{~mm}^{2}\right.$ size $)$ and the backscattered projectiles were detected with a Si solid-state detector located at a scattering angle of $170^{\circ}$. RBS spectra were acquired in channeling and random geometries to obtain additional information about the ion-induced amorphous layer. Additionally, morphological and compositional analysis were performed with a system equipped with a SPECS Phoibos 150 electron spectrometer designed to perform $\mathrm{x}$-ray photoelectron spectroscopy (XPS) analysis with a monochromatic $\mathrm{x}$-ray source, in combination with scanning electron (SEM) and Auger (SAM) microscopies with a FEI field-emission electron source and a secondary electron detector as part of the system. Using SAM, local chemical information was obtained by filtering the electron emission from the Fe LMM Auger transition. 

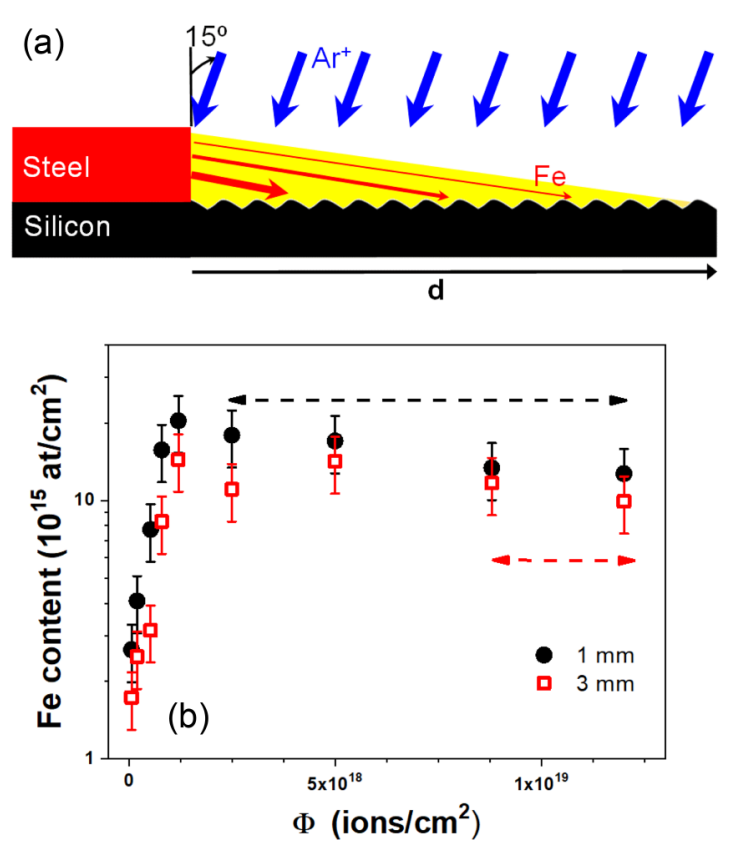

FIG. 1. (a) Experimental setup for medium-energy ion irradiation at $\theta=15^{\circ}$ with $\mathrm{Fe}$ codeposition from an adjacent steel plate. (b) Fe content measured by RBS at $d=1 \mathrm{~mm}$ and $d=3 \mathrm{~mm}$ for different ion fluences. The black/red dashed lines indicate the $\Phi$ range for which a ripple pattern is produced at $d$ values of 1 and $3 \mathrm{~mm}$, respectively.

\section{RESULTS AND DISCUSSION}

\section{A. Analysis of the metal content by RBS}

The experimental setup used in our experiments for the codeposition of metal is depicted in Fig. 1(a). Under such configuration, while the ions erode the $\mathrm{Si}$ surface, Fe atoms land on its surface coming from the sputtering produced by the ion beam on the metal plate. Obviously, less metal is codeposited on the irradiated silicon surface as the distance (d) from the metal clamp increases. As already observed in previous works [19,20,27], the metal content can play an important role in the pattern morphology. Therefore, we have quantified the residual $\mathrm{Fe}$ content at different irradiation times and two specific distances $(d=1$ and $3 \mathrm{~mm})$.

The results displayed in Fig. 1(b) show that the same qualitative behavior is observed for both $d$ values. Thus, the residual Fe content sharply increases until a maximum value is reached. Afterwards, a slow decrease regime takes place that could be compatible with a saturated one [28] if the error bars are considered. The Fe content in this final regime is, within experimental errors, slightly higher at $d=1 \mathrm{~mm}(4 \times$ $\left.10^{15} \mathrm{at} / \mathrm{cm}^{2}\right)$ than at $d=3 \mathrm{~mm}\left(3 \times 10^{15} \mathrm{at} / \mathrm{cm}^{2}\right)$. These values are almost two orders of magnitude smaller than those obtained with a similar setup but with the ion-beam incidence angle of $60^{\circ}$ [27]. However, it is close to those found at $d=5 \mathrm{~mm}$ in our former setup. The explanation of such difference in the late-stage Fe content is not straightforward, since several factors come into play. Not only $\theta$ is different, but also the ion-beam incidence angle on the vertical wall of the metal plate. In addition, the morphology of the irradiated Si target, which has a key role in the local ion-beam incidence

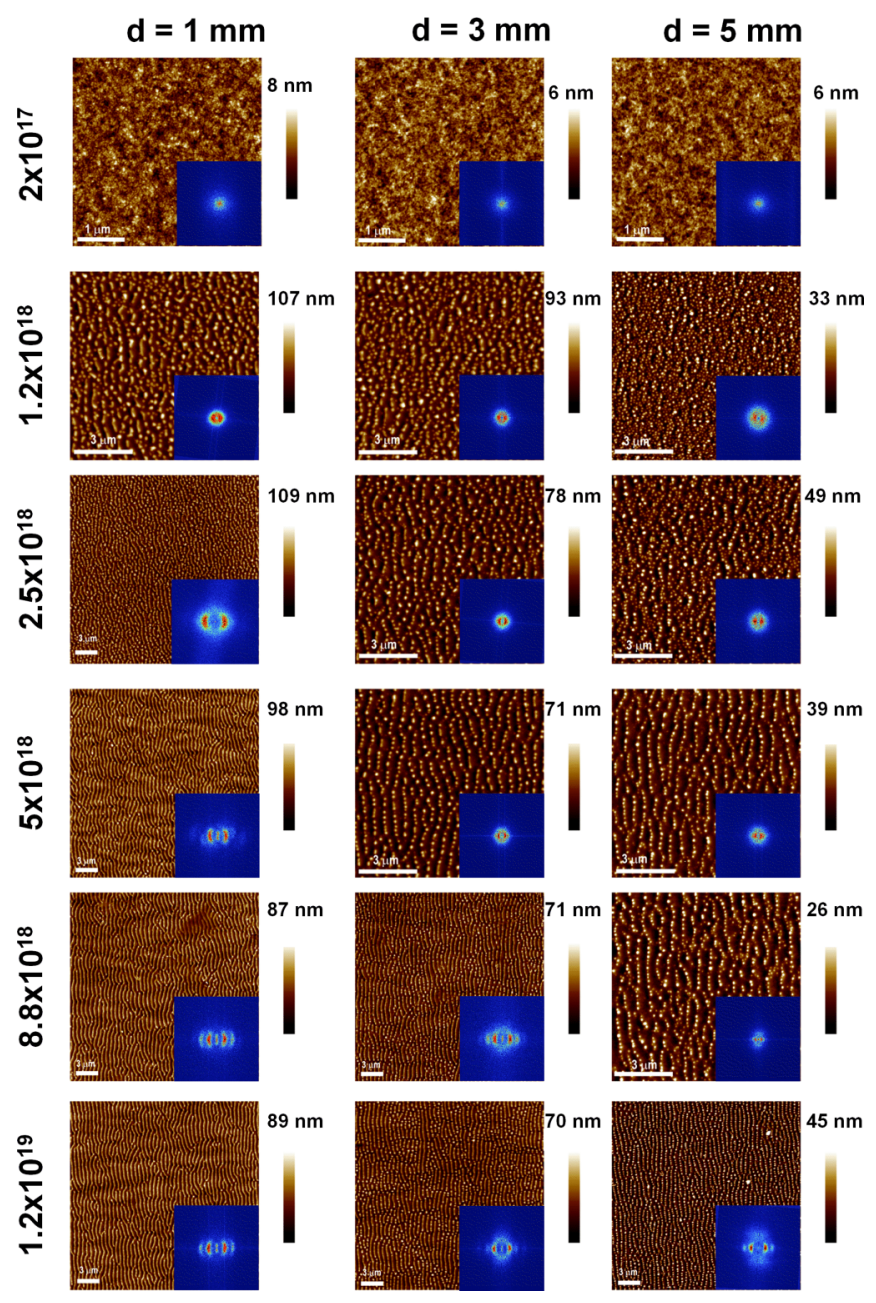

FIG. 2. AFM morphologies obtained at $d=1,3$, and $5 \mathrm{~mm}$ for the samples irradiated at increasing $\Phi$ values (given in ions $/ \mathrm{cm}^{2}$ ) The Fe flux comes from left to right whereas the ion-beam projected direction goes from right to left in the AFM images. In the insets are shown the corresponding FFT images.

angle and local surface sputtering rate, becomes very different for both systems [43].

\section{B. Morphological analysis of the ion-induced patterns}

Following the same scheme adopted in our former work [27], we have studied the pattern formation and development for three different $d$ values namely, at 1,3 , and $5 \mathrm{~mm}$. The characteristic morphologies are displayed in Fig. 2. As it was found for irradiations at $\theta=60^{\circ}$ [27], the pattern dynamics becomes appreciably slower as $d$ increases, i.e., as the metal content decreases. However, the morphological evolution for different $d$ values essentially follows similar stages. For very low $\Phi$ values, a slightly rough and disordered surface morphology is induced (top row of Fig. 2). As the irradiation proceeds, a sort of nanogranular structure appears. At these earlier stages (see the morphology at $d=5 \mathrm{~mm}$ for $\Phi=1.2 \times 10^{18}$ ions $/ \mathrm{cm}^{2}$ ), they do not display any ordering. Under further irradiation, these nanostructures can be referred to as nanodots, and they start to exhibit some alignment along the $y$ axis in the AFM images, i.e., along the perpendicular 

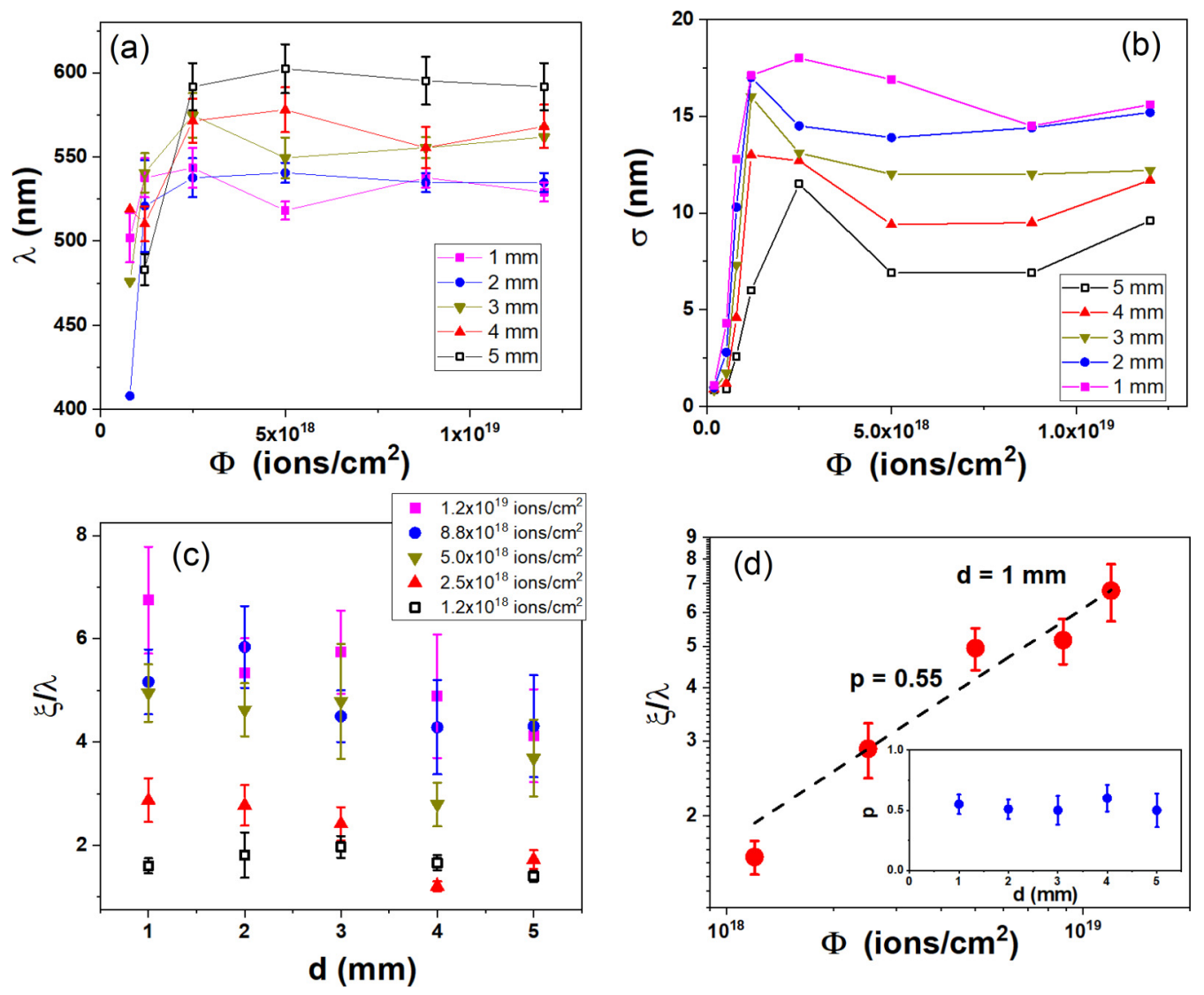

FIG. 3. Evolution of (a) $\lambda$ and (b) $\sigma$ with $\Phi$ for the different d values. Error bars in (b), in the 1.0-1.5 nm range, are not depicted for clarity

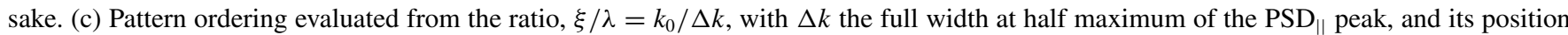
$k_{0}$, for different $d$ values and $\Phi$ values. (d) Logarithmic plot of $\xi / \lambda$ versus $\Phi$ for $d=1 \mathrm{~mm}$. The dashed line indicates the best linear fit with an exponent $p=0.55 \pm 0.08$. Inset: Plot of the exponent $p$ obtained for all $d$ values.

direction to the projection on the silicon surface of both the Fe flux and the $\mathrm{Ar}^{+}$ion beam. For longer irradiation stages, the dots along the vertical rows start to coalesce, resulting in rippled regions, which grow in length with $\Phi$. These rippled regions show a certain degree of curvature, a feature already reported in a similar system [28]. For the longest irradiation times and smaller $d$ values, the ripples become quite long (up to almost $18 \mu \mathrm{m}$ ) and straighter. Finally, the patterns beyond $5 \mathrm{~mm}$ either disappear for low fluences or, for the highest fluences, resemble the initial stages of pattern formation for short $d$ values (not shown).

The corresponding fast Fourier transform (FFT) images are displayed as insets. They show, for the three $d$ values, how from the initial featureless circular spot, the FFT evolves with increasing fluence to display first, for intermediate fluences, two main spots along the $x$ axis and at the highest fluences up to four spots, which indicates an enhancement of the pattern order with fluence. Likewise, it can be observed, particularly at some fluence values, how the FFT improves in terms of pattern-order features, for decreasing $d$ values. The issue of the pattern order will be addressed quantitatively below.

The above qualitative description made from the visual inspection of the AFM images is quantitatively confirmed in a detailed analysis shown in the Supplemental Material [44]. Briefly, this analysis shows that the induced ripples at $d=$ $1 \mathrm{~mm}$ improve morphologically with ion fluence, in terms of their orientation perpendicular to the Fe flux direction, length, aspect ratio, and straightness. In particular, as the ion fluence increases, the ripples become mostly oriented perpendicularly to the Fe flux direction and they run parallel to each other. In addition, and generally, the ripples show an asymmetric profile along the projected ion-beam direction, with a sharper slope at the shorter ripple side facing the incoming Fe flux than that observed at the wider ripple side facing the incoming ions. As noted in Ref. [44], since the induced nanostructures align themselves in a rowlike pattern along the perpendicular direction of both the ion-beam and the Fe flux projections, the $\mathrm{PSD}_{\|}$function becomes very useful to analyze the ripple morphology. Based on the analysis of the $\mathrm{PSD}_{\|}$peak width and the presence of harmonic peaks, it is qualitatively shown that the ripple pattern becomes more ordered as the ion fluence increases and $d$ decreases.

The $\mathrm{PSD}_{\|}$analysis is also useful to obtain the pattern wavelength $\lambda$. The evolution of $\lambda$ with $\Phi$ is plotted in Fig. 3(a) for several $d$ values. $\lambda$ increases initially with $\Phi$, during the dot regime, to attain a saturation value once a ripplelike pattern is formed (see the fixed peak position in Ref. [44], Fig. S4a). Thus, there is an initial coarsening that becomes interrupted at a certain fluence [45]; the smaller the $d$ value, the shorter its value. Noticeably, the saturated $\lambda$ value increases with $d$ value. In contrast, in the low-energy regime, Macko et al. did observe a significant variation of $\lambda$ with $d$ only for large $d$ 
values $(d>2.5 \mathrm{~mm})$ for which dot patterns were produced [18].

Another parameter that can be obtained from the AFM images is the surface roughness $\sigma$. Its evolution with ion fluence for different $d$ values is displayed in Fig. 3(b). The plots show a similar behavior for all $d$ values: an initial increase up to a maximum for $\Phi=(1.2-2.5) \times 10^{18} \mathrm{ions} / \mathrm{cm}^{2}$, followed by a slight decrease that, taking into account the corresponding error bars (not shown), could be compatible with a saturatedlike regime. In any case, $\sigma$ does not change so markedly in this fluence regime, and $\sigma$ decreases as $d$ increases. Noticeably, the maximum $\sigma$ is reached in all cases in the regime where dots dominate the surface morphology. This dependence differs from that found at low ion energies [19], where the higher roughness was observed for the asymptotic ripple regime. The maximum $\sigma$ tends to occur for higher $\Phi$ values as $d$ is larger. Moreover, for $d$ values in the 1-3-mm range, the maximum $\sigma$ is attained for conditions close to the maximum residual $\mathrm{Fe}$ content [Fig. 1(b)]. In fact, the dependence on ion fluence of both Fe content and $\sigma$ are akin.

The graphs in Figs. 3(a) and 3(b) for $\lambda$ and $\sigma$ are qualitatively similar since both reach a low decrease or saturatedlike regime after an initial steep increment. This behavior is opposite to that found at $60^{\circ}$ irradiation for the same sample setup [27], where both $\lambda$ and $\sigma$ increased for long irradiation times. There, large morphological features developed (with $\sigma$ values in the 150-200-nm range), and the presence of shadowing effects became important. It should be noted that in the present case, because of the relatively smooth morphology (without large slopes), shadowing effects can be discarded.

As noted above, as $\Phi$ increases and/or $d$ decreases (see Ref. [44], Figs. S3a and S3b) the $\mathrm{PSD}_{\|}$peaks become narrower, and the $\mathrm{PSD}_{\|}$curves present additional harmonic peaks at larger $k$ values. Both features are clear fingerprints of an increase in pattern ordering along the horizontal direction. In fact, it can be quantified by computing the ratio, $\xi / \lambda=$ $k_{0} / \Delta k$, where $\Delta k=1 / \xi$ is the full width at half maximum of the peak at position $k_{0}$ in the $\operatorname{PSD}_{\|}[42,46]$. $\xi$ is referred to as the ordered domain size. Therefore, the ratio $\xi / \lambda$ is a normalized measure of the order. Since pattern ordering is improved as the $\mathrm{PSD}_{\|}$peak becomes sharper, i.e., narrower, it should lead to an increase of $\xi / \lambda$. This is indeed the case for all $d$ values [Fig. 3(c)], since $\xi / \lambda$ increases with $\Phi$ in all cases. From this figure, it is also clear that at smaller $d$ values, the order is enhanced since larger values of $\xi / \lambda$ are achieved for a given ion fluence. Moreover, the pattern-order enhancement rate can be obtained by plotting $\xi / \lambda$ versus $\Phi$ for each $d$ value. As an example, Fig. 3(d) shows such plot for the $d=1-\mathrm{mm}$ case. From the logarithmic plot, $\xi / \lambda$ follows a power law $\left(\xi / \lambda \sim \Phi^{p}\right)$. Thus, $p$ describes the pattern-order enhancement rate, since a higher value of $p$ means faster pattern ordering. For $d=1 \mathrm{~mm}, p$ is equal to $0.55 \pm 0.08$. When this analysis is repeated for all $d$ values, it results [see inset of Fig. 3(d)] that a similar ordering enhancement rate with $\Phi$, within the error bars, is obtained. It should be remarked that, for a fixed $\Phi$ value, the larger the $d$ value, the less ordered is the pattern, but the pattern order improves with $\Phi$ at the same rate for different $d$ values. This implies that while the pattern order does depend on $d$ (i.e., on the Fe flux), its enhancement rate with ion fluence does not.
It is interesting to note that the order enhancement with $\Phi$ is a feature not found in low-energy IBS patterning of silicon surfaces under clean conditions [47]. In particular, for a bombardment angle moderately above the threshold angle $\left(\theta=63^{\circ}\right)$ the pattern becomes more disordered with $\Phi$. This behavior is even emphasized when the angle becomes larger $\left(\theta=75^{\circ}\right)$. On the other hand, in our previous work [27], where silicon surfaces were patterned by IBS with $\mathrm{Fe}$ codeposition, the patterns became rather disordered with $\Phi$, likely because of the strong shadowing effects. In contrast, dot patterns produced by low-energy IBS with isotropic Fe codeposition displayed increased order with $\Phi[46,48]$. Therefore, IBS with metal codeposition can lead to enhanced ordered patterns under appropriate conditions or configurations. It should be noted that, as pointed out by Bradley [37], in the first experiments when the codeposition of impurities in the IBS patterning was unnoticed in some cases exceptionally highly ordered patterns were produced [48-50].This fact implies that by deepening the knowledge of the mechanisms of pattern ordering through well controlled and designed experiments the use of IBS as a nanofabrication tool can be further enhanced.

From this morphological analysis, we can compare the pattern characteristics with those obtained in our previous study with medium-energy ions as well as with those performed with low-energy ions, all with a similar setup [18-20,26,28,30]. Liu et al. reported the nanostructuring of a $\mathrm{SiO}_{2}$ surface by IBS with $\mathrm{Al}$ codeposition only for ion incidence angles equal to or larger than $40^{\circ}$ [30]. In contrast, in the works from the group of Hofsäss with $5 \mathrm{keV} \mathrm{Xe} \mathrm{Xe}^{+}$ irradiation of silicon at normal incidence, and a tilted metal plate, the surface becomes nanopatterned due to the metal codeposition [19,28]. Moreover, the pattern evolution is akin to that found in our system: initially, the surface remains flat until nanodots or grains are formed. Afterwards, the dots become aligned along the perpendicular direction to the projection of the incoming Fe flux. At later stages, the ripples become longer and straighter. Furthermore, the ripple patterns are produced for relatively large $\mathrm{Fe}$ contents, from 5.4 to $13.1 \times 10^{15} \mathrm{Fe} / \mathrm{cm}^{2}$. However, the better ripple patterns are obtained for $5.4 \times 10^{15} \mathrm{Fe} / \mathrm{cm}^{2}$ [19], whereas in our case, the ripple patterns with the highest order are achieved for contents close to $10^{16} \mathrm{Fe} / \mathrm{cm}^{2}$. The most evident differences between the low-energy and our medium-energy ripple patterns are the enhanced order as well as the larger wavelength, i.e., larger than $500 \mathrm{~nm}$, obtained in the present study, compared to the low-energy case, where the wavelength can be estimated to be around $120 \mathrm{~nm}$. With respect to the first difference, it is likely due to the smaller maximum ion fluence employed at low energy, which is one order of magnitude smaller than the one used in our system. Regarding the second difference, it has to be associated with the higher ion energy used in this study, which affects both the wavelength and the roughness, as it was already reported for the case of nanodots produced by IBS with metal codeposition [51]. However, the increment in roughness is not so high, $15 \mathrm{~nm}$ for medium-ion energy compared to $10 \mathrm{~nm}$ for the low-energy case.

It is interesting to analyze our present data in the framework of the model by Bradley for pattern formation under IBS with metal codeposition [37]. In this model, the key mechanism triggering the pattern development is the com- 
pound formation between the impurity and the target elements, i.e., the silicide formation in the current case. The relevance of silicide formation for pattern development in systems similar to ours has been assessed experimentally $[24,26-28,31-33,51]$. Thus, we can view our system in the light of this model.

We have to note that, by working at $15^{\circ}$ incidence, the Carter-Vishnyakov (CV) effect produces a surface current that has a clear smoothing effect on the irradiated surface [52], which is the scenario contemplated in Bradley's work too. This fact is important because, on the contrary, the CV effect tends to destabilize the bombarded surface if the incidence angle is larger than $45^{\circ}$ [53], which applies to our former study [27]. Due to the specific experimental setup, it is possible to compare qualitatively the experimental findings with the predictions of the model.

Firstly, the ripples produced in our experiments have wave vectors that are aligned with the projected direction of the incident $\mathrm{Fe}$ flux as predicted by the model. Secondly, the model highlights the influence of the metal atom-to-ion arrival rate ratio, $j$, on the pattern wavelength. In our system, we cannot compute this ratio, since we do not have the required knowledge about the Fe flux on the surface, which is coupled with the ion bombardment and the setup geometry. As the ion beam impinges on the $\mathrm{Fe}$ plate with an incidence angle of $75^{\circ}$ with respect to its normal, the corresponding sputtering yield is rather high, i.e., around 24 atoms/ion according to SRIM simulations (not shown). Therefore, a significant flux of $\mathrm{Fe}$ emerges from the plate, which is directed towards the silicon target surface and, obviously, it decreases as $d$ increases. On the other hand, the incoming ion rate is homogeneous across the bombarded surface. Therefore, we can assume that for increasing $d$ values $j$ decreases, which is supported by the smaller Fe content measured by RBS for larger distances (see Fig. 1).

In Bradley's model, pattern formation occurs for a $j$ value larger than a threshold value $j^{*}$. As we detect pattern formation up to $d=7 \mathrm{~mm}$, we can state that we are obviously above this value. According to the model, $\lambda$ should decrease as $j$ increases following the relationship: $\lambda \propto\left(j-j^{*}\right)^{-0.5}$. This dependence is difficult to assess quantitatively due to the lack of knowledge of $j$ and $j^{*}$. However, from the $\lambda$ values derived from the $\mathrm{PSD}_{\|}$curves, we can plot how $\lambda$ changes as $d(j)$ increases (decreases); see Fig. 4.

This plot shows that $\lambda$ is increasingly higher as $d$ increases, i.e., as $j$ decreases, which is in agreement with the model [37]. It is worth to note that this trend is followed for all $\Phi$ values sampled. In principle, despite the difficulty in knowing precisely how $j$ decreases with $d$, we can assume that, in a rough approximation, $j \propto 1 / d^{2}$. Thus, we have attempted to fit the data of Fig. 4 with the following equation:

$$
\lambda=A+B /\left[\left(10^{6} / d\right)^{2}-C\right]^{0.5},
$$

where we have used $j=10^{12} / d^{2}$, with $d$ in nm, with $A, B$, and $C$ as the fitting parameters. It is observed that for the three larger fluences, where the patterns are better developed, Eq. (1) fits adequately well the experimental data since for small $d$ values, $\lambda$ depends linearly on $d$, whereas for larger distances (lower $j$ ) it starts to diverge to larger values.

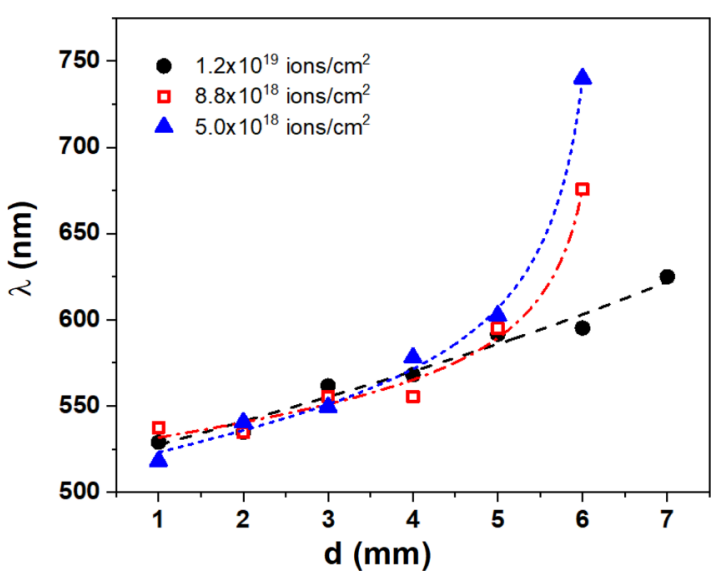

FIG. 4. Plot of $\lambda$ for the different $d$ values and higher $\Phi$ values. Note that higher $d$ values imply a smaller Fe flux $j$. The solid lines represent the best fit of Eq. (1) for each $\Phi$ value.

Finally, it should be commented that the incoming Fe flux, both in terms of magnitude and directionality, plays a certain role in the pattern dynamics because, from Fig. 1(b), it is seen that the Fe content has practically reached the steady state after $1 \mathrm{~h}$ of irradiation. In fact, Macko et al. [20] determined the relevance of the angle between both, Fe and ions, flux directions on the pattern formation. In this sense, it should be commented that the direct patterning by IBS under clean conditions of an iron silicide target has been unsuccessful when it has been attempted [54].

\section{Lateral and in-depth distribution of $\mathrm{Fe}$ in the evolving pattern: Compositional patterning}

As noted above, Bradley's model is based on a key mechanism that triggers the pattern, which is the compound formation between the impurity and the target elements, i.e., the silicide formation in our case. This mechanism can be enhanced for medium-energy IBS due to the promotion of ion-beam mixing effects [55] or the presence of thermal spikes [56,57]. Besides, energetic ion impacts can induce a higher mobility of lighter elements ( $\mathrm{Si}$ ) [58]. In our system, this is very likely due to the pattern morphology dependence on the $\mathrm{Fe}$ content as well as to the fact that, without simultaneous $\mathrm{Fe}$ codeposition, no pattern is induced on Si by IBS at $15^{\circ}$. Importantly, Bradley's model predicts that the silicide content should be higher on top of the ripples than at the valleys. Therefore, in order to assess this fact in our system, we have used a series of techniques to determine whether a compositional pattern is formed as well as its eventual correlation with the morphological one.

The top panel in Fig. 5 shows a cross-sectional bright-field TEM image of the sample produced after $\Phi=8.8 \times 10^{18}$ ions $/ \mathrm{cm}^{2}$. The location corresponds approximately to $d=$ $1 \mathrm{~mm}$. Four ripple morphologies can be seen in the image. Clearly, the amorphous surface protrusions show a darker mass-thickness contrast, indicating a higher-mass material in these regions compared to the surrounding amorphized silicon. About $100 \mathrm{~nm}$ below the surface, the Si substrate remains single crystalline. In the middle panel, a higher-magnified image displays two ripple structures in more detail. This image 


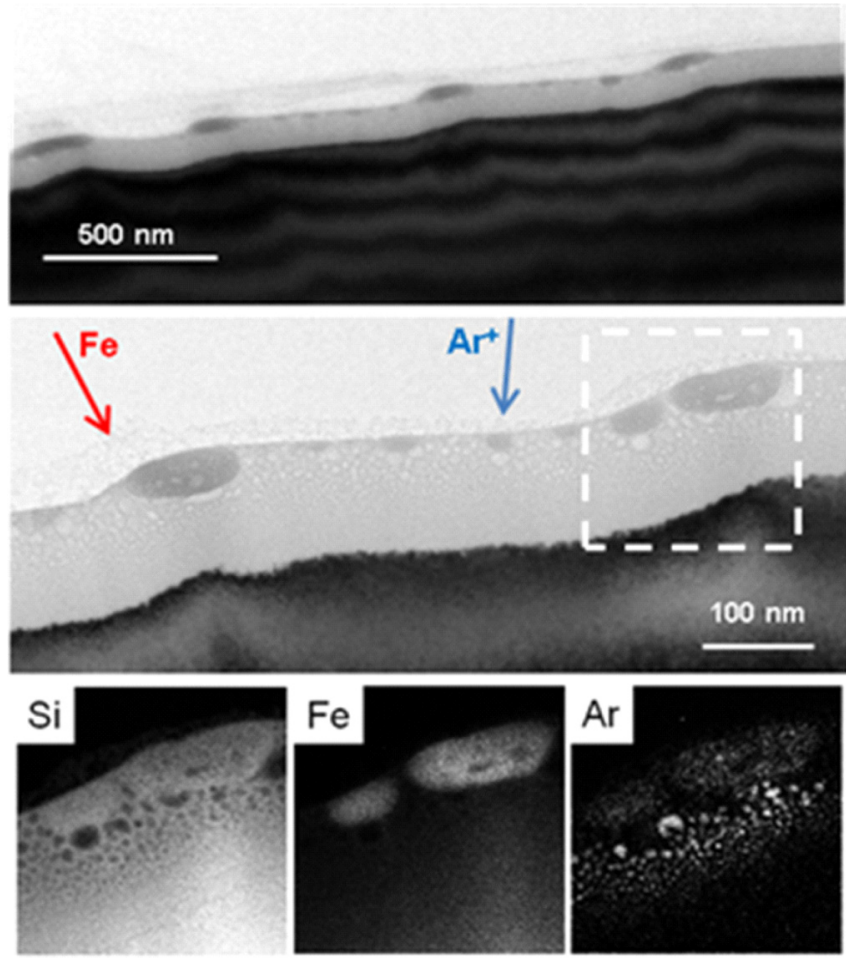

FIG. 5. Top: bright-field TEM image of the sample irradiated with $\Phi=8.8 \times 10^{18}$ ions $/ \mathrm{cm}^{2}$ at $d \sim 1 \mathrm{~mm}$ showing the cross section of four ripple nanostructures (darker zones). Middle: bright-field TEM image showing in detail two ripple structures. The $\mathrm{Ar}^{+}$and Fe flux directions are indicated with arrows. The image was taken with slight underfocus in order to highlight the presence of Ar bubbles produced by the $40-\mathrm{keV} \mathrm{Ar}^{+}$bombardment. Bottom: Highermagnification EFTEM images from the area within the dashed square in the middle panel showing the distribution of $\mathrm{Si}, \mathrm{Fe}$, and $\mathrm{Ar}$ atoms.

was taken at slight underfocus conditions to clearly show the Ar bubble distribution caused by the ion implantation. It is evident that the bubbles are larger when closer to the surface as it was reported for medium-energy IBS of Si surfaces under impurity-free conditions [36]. It is worth to note that the larger Fe-rich regions are found at the surface protuberances facing the incoming Fe flux, which agrees with the model by Bradley [37]. In the bottom insets of Fig. 5, higher-magnification energy-filtered (EF)TEM images of the right ripple (marked by the dashed square) show the distributions of $\mathrm{Si}, \mathrm{Fe}$, and $\mathrm{Ar}$ for the selected area. It is evident that the ripple structures are Fe-rich regions in comparison with other zones of the surface. In addition, smaller Fe-rich aggregates are also detected on the wider slope of the ripple structure. It should be noticed that Ar bubbles are also found within the Fe-rich ripple regions. These data prove that the ripple structures observed by AFM clearly correspond to rather localized Fe-rich structures where $\mathrm{Si}$ atoms are also present. However, the amorphous character of these regions hampers the possibility to identify them by EFTEM as crystalline iron silicide-rich locations. For that purpose, we performed XPS and SEM/SAM experiments.

Figure 6(a) displays the XPS Fe $2 p$ core-level emission excited with monochromatic $\mathrm{Al} K \alpha$ radiation for the sample (a)

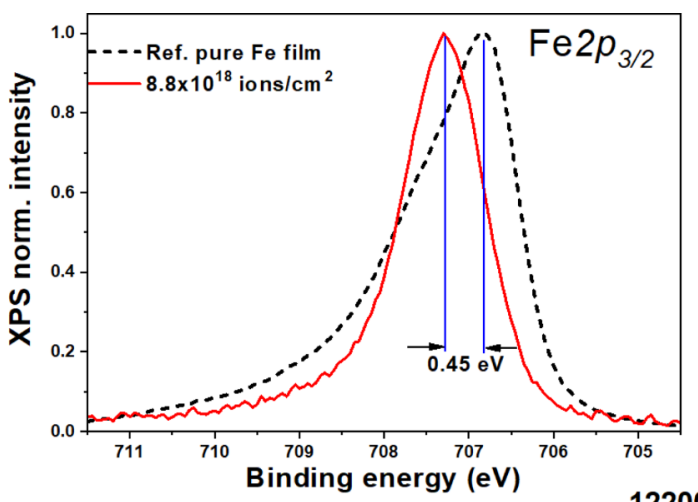

(b)

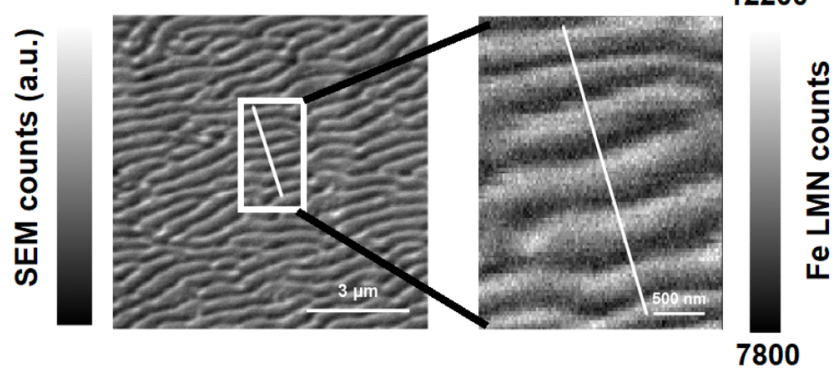

(c)

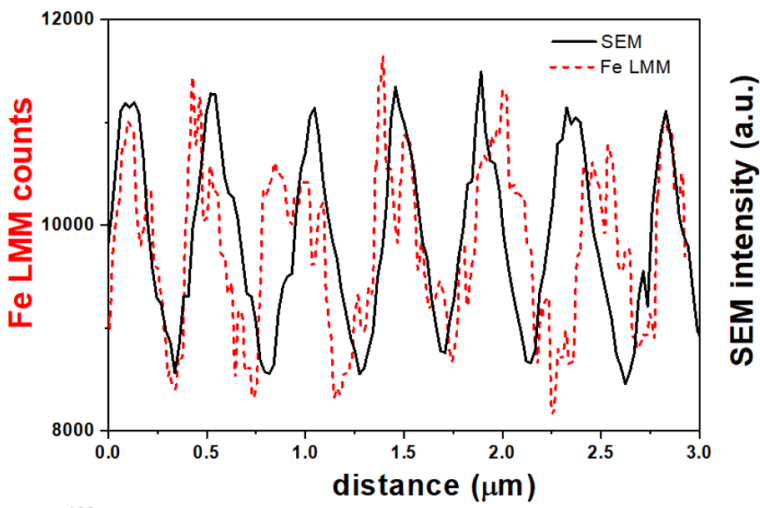

(d)

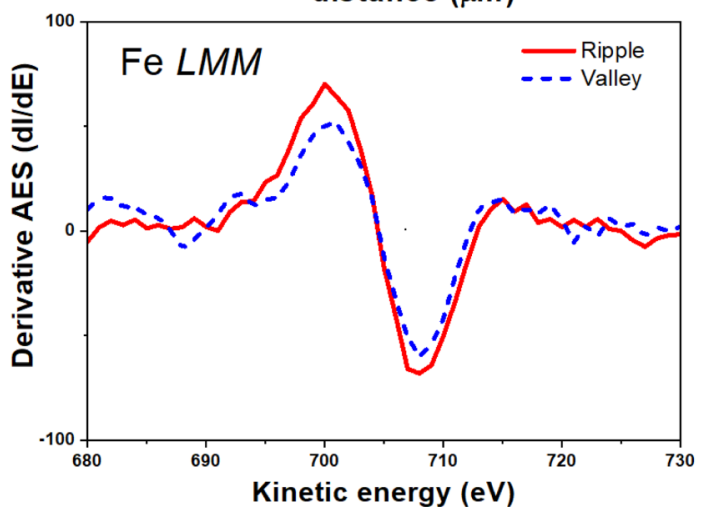

FIG. 6. (a) XPS spectra of the Fe $2 p$ core level of the sample irradiated with $8.8 \times 10^{18}$ ions $/ \mathrm{cm}^{2}$ and the reference Fe film; the chemical shift with respect to the Fe reference signal is indicated. (b) SEM image obtained on a rippled region (left) and SAM image (right) from the region highlighted in the SEM image recorded by filtering the electrons emitted from the Fe LMM Auger transition $(\sim 705 \mathrm{eV})$ and after background subtraction $(\sim 725 \mathrm{eV})$. The grayscale corresponds to an increase in the number of counts of $\sim 25 \%$ (from black to white). (c) Line scans across SEM and SAM images marked in (b). (d) Fe LMM AES spectra in derivative mode acquired at two positions on ripple and valley regions. 

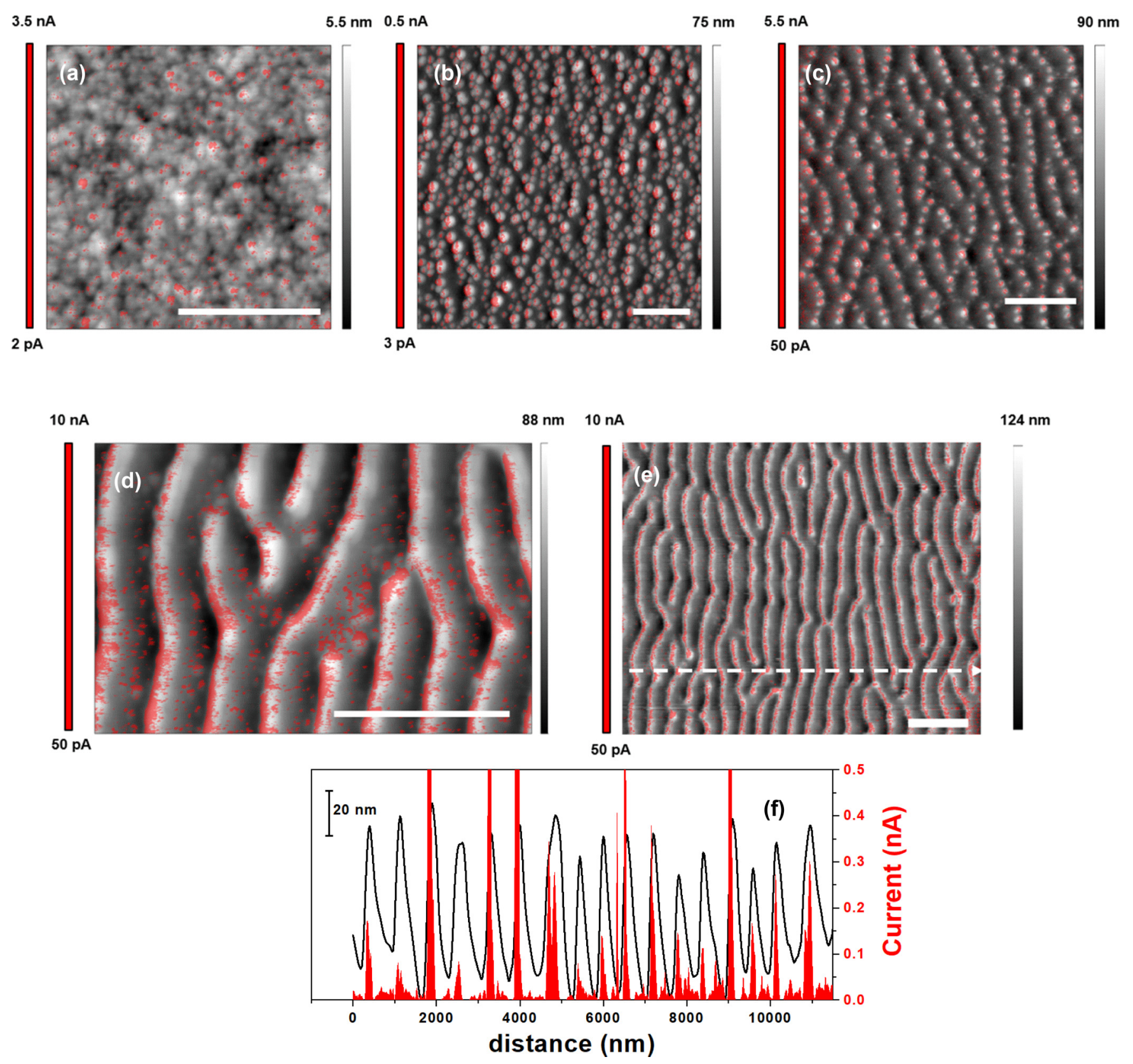

FIG. 7. Current-sensing signal above the threshold (red) overlapped on the morphological AFM image (grayscale) for different stages of the pattern development at $d \sim 1 \mathrm{~mm}$ : (a) $1.2 \times 10^{18}$ ions $/ \mathrm{cm}^{2}$; (b) $2.5 \times 10^{18}$ ions $/ \mathrm{cm}^{2}$; (c) $5 \times 10^{18}$ ions $/ \mathrm{cm}^{2}$; (d) $8.8 \times 10^{18}$ ions $/ \mathrm{cm}^{2}$; (e) $1.2 \times 10^{19}$ ions $/ \mathrm{cm}^{2}$. (f) Cross section of the corresponding topographical (black line) and current (red filled plot) images of (e) along the dashed line. (The horizontal bars correspond to $2 \mu \mathrm{m}$.) The Fe flux comes from left to right whereas the ion-beam projected direction goes from right to left in the AFM images. In the CS-AFM image, only those data points displaying a current value equal or higher than the threshold value (bottom value in the vertical scale bar) are marked in red.

irradiated with $8.8 \times 10^{18}$ ions $/ \mathrm{cm}^{2}$ in comparison with that of a reference pure Fe film. As readily seen, there is a clear difference in both line shape and peak energy position in the spectra. On the one hand, the irradiated sample shows a symmetric line shape and narrower width; on the other, there exists a chemical shift of $\sim 0.45 \mathrm{eV}$ towards higher binding energy values with respect to the metal reference. These results together are characteristic of Fe-silicide formation and allow us to ascribe its presence in agreement to results already reported in a previous work [27]. In order to study correlations between surface morphology and local chemical composition
SAM experiments were carried out, which further support that the ripple structures are Fe-rich regions. On the one side, Fig. 6(b) (left) shows the corresponding morphology extracted from the total secondary electron emission where the ripple pattern is clearly resolved, and, on the other, Fig. 6(b) (right) displays the corresponding SAM image of the selected zone marked with a box in Fig. 6(b) (left). In this case, the SAM image is recorded by analyzing the electron emission from the Fe LMM Auger transition and, hence, a higher intensity corresponds to a higher $\mathrm{Fe}$ content in the sample. In addition, line scans taken across the same location in the SEM 
and SAM images are shown in Fig. 6(c), which reveal that both the morphological and chemical signals are nicely in phase. In particular, local chemical analysis was obtained by the quantification of Fe LMM Auger electron spectroscopy (AES) spectra in derivative mode acquired at two positions on ripple and valley regions as shown in Fig. 6(d). Note that the intensity of the signal from the ripple region is enhanced by $\sim 25 \%$ with respect to that of the valley one, indicating a higher Fe content at the ripple peaks. It is worth mentioning that these measurements were performed on several ripple and valley spots being representative of those shown in Fig. 6(d). These measurements underline the SAM results in which the brighter structures correspond to a higher Fe signal and, correspondingly, also in the ripples of Fig. 6(b) (left). It should be noted that it was possible to obtain this information due to the large ripple wavelength produced by the medium-energy ion irradiation. Probably, the SAM technique would not be able to obtain it for the ripple patterns produced by low-energy IBS.

In order to confirm these results with higher lateral resolution, we employed the CS-AFM technique. In this mode, we obtain simultaneously information on the surface morphology and its local conductive response. This study is based on our previous work [27], where we demonstrated that the Fe-rich regions yielded higher currents than $\mathrm{Fe}$-poor ones. In this case, as well as in the previous one [27], we have not identified the silicides present in the patterned surfaces. However, there are silicides with different stoichiometry as well as different phases, some of them semiconducting and others metallic $[59,60]$ that could explain the enhanced current measured on the silicide-rich regions with respect to the Fe-poor and amorphized silicon regions. Figure 7 shows the evolution of the morphology (grayscale) and the simultaneous conductivity mapping. For the latter only the values above a certain threshold are superimposed in red on the morphological image to help locating the silicide-rich spots and to establish the correlation between both patterns. It is worth noting that this threshold value is higher for the three larger fluences.

Figure 7(a) corresponds to the very early stages of irradiation with Fe codeposition. In this regime, a disordered granular morphology is observed without any hint of patterning. The red spots seem to be located rather randomly on the surface. In addition, the sensed currents are extremely low (below $1 / 10$ of $\mathrm{nA}$ ) in agreement with the small metal content [Fig. 1(b)]. At $\Phi=1.2 \times 10^{18}$ ions $/ \mathrm{cm}^{2}$, clear nanodot structures, without significant ordering, have been formed that yield higher currents than the surrounding flatter areas [Fig. 7(b)]. The same behavior is found for $\Phi=2.5 \times 10^{18}$ ions $/ \mathrm{cm}^{2}$ [Fig. 7(c)], when the nanodots tend to organize along vertical rows. Finally, for the latter stages where a clear and ordered ripple pattern has developed [Figs. 7(d) and 7(e)], the ripples are more conductive than the flatter (darker) regions. The high current regions are localized mainly on the sharpest-sloped side of the ripples, i.e., that side facing the incoming Fe flux. This is shown in the profiles depicted in Fig. 7(f) along the dashed line of Fig. 7(e). Clearly, the highest local current peaks take place at the sharpest ripple slopes. This fact agrees with the data obtained by TEM where Fe-rich regions were identified on the ripple features (Fig. 5). It is interesting to observe Fig. 7(d) in detail, since, besides the evident ripple features, some small granular structures are also observed on the lowest-slope side of the ripples. Many of these nanograins also yield higher currents than the flat regions, which indicates that they are also Fe-rich zones as also evidenced in TEM analysis. However, these regions, which should have a smaller sputtering rate than the Fe-poor ones, do not evolve into higher features as it is the case of the marked ripple structures. These results were confirmed by KFM measurements (see Ref. [44], Fig. S5). It should be noted that this enhanced Fe content at the ripple peak corresponds with the prediction of the Bradley model (see Fig. 1 in Ref. [37]).

\section{CONCLUSIONS}

The results presented in this study evidence that nearnormal medium-energy ion irradiation with oblique metal codeposition can be used to induce pattern formation on $\mathrm{Si}(100)$ surfaces with enhanced order, comprising the formation of relatively long ripple nanostructures (i.e., close to $18 \mu \mathrm{m}$ ) running parallel to each other and oriented along the perpendicular to the Fe flux projection direction. These features are more marked for long irradiation times and at locations closer to the metal source, i.e., where the Fe flux is higher. In particular, a pattern transition from randomly distributed nanodots, spaced nanodot chains, to well-developed ripple/nanowirelike structures can be obtained by tuning the ion fluence and Fe incorporation rate. Whereas the pattern order for a given ion fluence is higher for larger Fe flux, the rate of order enhancement with ion fluence does not depend on this magnitude. We have analyzed our data in the framework of the main model dealing with ion-induced pattern formation under metal codeposition. Thus, we have verified that the pattern wavelength decreases with the Fe flux, with a behavior consistent with this model. Moreover, the ripples are oriented along the perpendicular direction to that of the incoming $\mathrm{Fe}$ flux, in agreement with the model. A key assumption of the model refers to the formation of a compound between the target $(\mathrm{Si})$ atoms and the metal ones $(\mathrm{Fe})$; in our case this compound is a silicide. Accordingly, we have used several characterization tools to prove that the prominent morphological features (dots or ripples) are silicide-rich regions. Furthermore, we have verified by different local morphological and compositional techniques that there is a compositional pattern coupled with the morphological one, as also predicted in the model. It is worth to note that the above analysis become more accessible due to the large pattern dimensions produced by medium-energy IBS in comparison with those obtained by low-energy IBS. Finally, it is worth noting that near-normal IBS with oblique metal codeposition can lead to more ordered ripple patterns than either those obtained under similar conditions but with ion-beam incidence angles above the threshold value, or those obtained without metal codeposition. In particular, we hope that the analysis of the pattern order may motivate further theoretical study since it is an issue that remains to be addressed. Furthermore, the advance in the understanding of the mechanisms behind the pattern-order enhancement for IBS with metal codeposition, through the design of well-controlled experimental settings, could enable the production of ordered patterns over large areas and short irradiation times. 


\section{ACKNOWLEDGMENTS}

This research is supported by Spanish MINECO (Grants No. MAT2016-80394-R and No. MAT2017-85089-C2-1R) and the TRANSNANOAVANSENS program (Grant No. S2018/NMT-4349) from the Comunidad de Madrid. A.R.C. acknowledges Ramón y Cajal program (under Contract No. RYC-2015-18047). F.J.P. is thankful for financial support by A.E. Consejo Superior de Investigaciones Científicas (under Grant No. CSIC-2019AEP150). The use of HZDR Ion Beam Center TEM facilities is gratefully acknowledged.
[1] J. Muñoz-García, L. Vázquez, R. Cuerno, J. A. Sánchez-García, M. Castro, and R. Gago, in Toward Functional Nanomaterials, edited by Z. M. Wang (Springer, Dordrecht, 2009).

[2] C. S. Madi, H. B. George, and M. J. Aziz, J. Phys.: Condens. Matter 21, 224010 (2009).

[3] C. S. Madi, E. Anzenberg, K. F. Ludwig, and M. J. Aziz, Phys. Rev. Lett. 106, 066101 (2011).

[4] M. Castro and R. Cuerno, Appl. Surf. Sci. 258, 4171 (2012).

[5] S. A. Norris, Phys. Rev. B 85, 155325 (2012).

[6] M. Castro, R. Gago, L. Vázquez, J. Muñoz-García, and R. Cuerno, Phys. Rev. B. 86, 214107 (2012).

[7] A. Moreno-Barrado, M. Castro, R. Gago, L. Vázquez, J. Muñoz-García, A. Redondo-Cubero, B. Galiana, C. Ballesteros, and R. Cuerno, Phys. Rev. B 91, 155303 (2015).

[8] S. A. Norris, J. C. Perkinson, M. Mokhtarzadeh, E. Anzenberg, M. J. Aziz, and K. F. Ludwig, Sci. Rep., 7, 2016 (2017).

[9] M. P. Harrison, D. A. Pearson, and R. M. Bradley, Phys. Rev. E 96, 032804 (2017).

[10] H. Hofsäss and O. Bobes, Appl. Phys. Rev. 6, 021307 (2019).

[11] J. Muñoz-García, R. Cuerno, and M. Castro, Phys. Rev. B 100, 205421 (2019).

[12] M. Teichmann, J. Lorbeer, B. Ziberi, F. Frost, and B. Rauschenbach, New J. Phys. 15, 103029 (2013).

[13] S. Rusponi, G. Costantini, C. Boragno, and U. Valbusa, Phys. Rev. Lett. 81, 2735 (1998); 81, 4184 (1998).

[14] U. Valbusa, C. Boragno, and F. Buatier de Mongeot, J. Phys.: Condens. Matter 14, 8153 (2002).

[15] H. Hofsäss and K. Zhang, Appl. Phys. A: Mater. Sci. Process. 92, 517 (2008).

[16] J. A. Sánchez-García, R. Gago, R. Caillard, A. RedondoCubero, J. A. Martin-Gago, F. J. Palomares, M. Fernández, and L. Vázquez, J. Phys.: Condens. Matter 21, 224009 (2009).

[17] G. Ozaydin-Ince and K. F. Ludwig, Jr., J. Phys.: Cond. Matter 21, 224008 (2009).

[18] S. Macko, F. Frost, B. Ziberi, D. F. Förster, and T. Michely, Nanotechnology 21, 085301 (2010).

[19] K. Zhang, M. Brötzmann, and H. Hofsäss, New J. Phys. 13, 013033 (2011).

[20] S. Macko, F. Frost, M. Engler, D. Hirsch, T. Höche, J. Grenzer, and T. Michely, New J. Phys. 13, 073017 (2011).

[21] K. Zhang, O. Bobes, and H. Hofsäss, Nanotechnology 25, 085301 (2014).

[22] G. Ozaydin, A. S. Özcan, Y. Wang, K. F. Ludwig, H. Zhou, R. L. Headrick, and D. P. Siddons, Appl. Phys. Lett. 87, 163104 (2005)

[23] C. Teichert, C. Hofer, and G. Hlawacek, Adv. Eng. Mater. 8, 1057 (2006).

[24] J. Muñoz-García, L. Vázquez, M. Castro, R. Gago, A. Redondo-Cubero, A. Moreno-Barrado, and R. Cuerno, Mater. Sci. Eng. R. Rep. 86, 1 (2014).
[25] H. Hofsäss and K. Zhang, Nucl. Instrum. Methods Phys. Res. B 267, 2731 (2009).

[26] K. Zhang, M. Brötzmann, and H. Hofsäss, AIP Adv. 2, 032123 (2012).

[27] A. Redondo-Cubero, R. Gago, F. J. Palomares, A. Mücklich, M. Vinnichenko, and L. Vázquez, Phys. Rev. B 86, 085436 (2012).

[28] H. Hofsäss, K. Zhang, A. Pape, O. Bobes, and M. Brötzmann, Appl. Phys. A 111, 653 (2013).

[29] S. K. Vayalil, A. Gupta, and S. V. Roth, Appl. Phys. A 123, 225 (2017).

[30] Y. Liu, D. Hirsch, R. Fechner, Y. Hong, S. Fu, F. Frost, and B. Rauschenbach, Appl. Phys. A 124, 73 (2018).

[31] M. Engler, F. Frost, S. Müller, S. Macko, M. Will, R. Feder, D. Spemann, R. Hübner, S. Facsko, and T. Michely, Nanotechnology 25, 115303 (2014).

[32] R. Gago, A. Redondo-Cubero, F.J. Palomares, and L. Vazquez, Nanotechnology 25, 415301 (2014).

[33] B. Khanbabaee, D. Lützenkirchen-Hecht, R. Hübner, J. Grenzer, S. Facsko, and U. Pietsch, J. Appl. Phys. 116, 024301 (2014).

[34] B. Moon, S. Yoo, J.-S. Kim, S. J. Kang, J. Muñoz-García, and R. Cuerno, Phys. Rev. B 93, 115430 (2016).

[35] K. S. Lloyd, I. L. Bolotin, M. Schmeling, L. Hanley, and I. V. Veryovkina, Surf. Sci. 652, 334 (2016).

[36] T. K. Chini, D. P. Datta, and S. R. Bhattacharyya, J. Phys.: Condens. Matt. 21, 224004 (2009).

[37] R. M. Bradley, Phys. Rev. B 87, 205408 (2013).

[38] C. Teichert and I. Beinik, in Scanning Probe Microscopy in Nanoscience and Nanotechnology, edited by B. Bhushan (Springer, Berlin, 2011), Vol. 2, Chap. 23.

[39] D. Nečas and P. Klapetek, Cent. Eur. J. Phys. 10, 181 (2012).

[40] C. A. Schneider, W. S. Rasband, and K. W. Eliceiri, Nat. Methods 9, 671 (2012).

[41] Z. Püspöki, M. Storath, D. Sage, and M. Unser, Adv. Anat. Embryol. Cell Biol. 219, 69 (2016).

[42] Y.-P. Zhao, G.-C. Wang, and T.-M. Lu, Characterization of Amorphous and Crystalline Rough Surfaces: Principles and Applications (Academic Press, San Diego, 2001).

[43] G. Carter, J. Appl. Phys. 85, 455 (1999).

[44] See Supplemental Material at http://link.aps.org/supplemental/ 10.1103/PhysRevB.102.075423 for a detailed AFM and KFM characterization of the irradiated surfaces.

[45] J. Muñoz-García, R. Gago, R. Cuerno, J. A. Sánchez-García, A. Redondo-Cubero, M. Castro, and L. Vázquez, J. Phys.: Condens. Matter 24, 375302 (2012).

[46] R. Gago, L. Vázquez, O. Plantevin, T. Metzger, J. MuñozGarcía, R. Cuerno, and M. Castro, Appl. Phys. Lett. 89, 233101 (2006).

[47] M. Engler, S. Macko, F. Frost, and T. Michely, Phys. Rev. B 89, 245412 (2014). 
[48] R. Gago, L. Vázquez, R. Cuerno, M. Varela, C. Ballesteros, and J. M. Albella, Appl. Phys. Lett. 78, 3316 (2001).

[49] B. Ziberi, F. Frost, T. Höche, and B. Rauschenbach, Phys. Rev. B 72, 235310 (2005).

[50] B. Ziberi, M. Cornejo, F. Frost, and B. Rauschenbach, J. Phys.: Condens. Matter 21, 224003 (2009).

[51] A. Redondo-Cubero, B. Galiana, K. Lorenz, F. J. Palomares, D. Bahena, C. Ballesteros, I. Hernández-Calderón, and L. Vázquez, Nanotechnology 27, 444001 (2016).

[52] G. Carter and V. Vishnyakov, Phys. Rev. B 54, 17647 (1996).

[53] B. Davidovitch, M. J. Aziz, and M. P. Brenner, Phys. Rev. B 76, 205420 (2007).
[54] R. Gago, M. Jaafar, and F.J. Palomares, J. Phys.: Condens. Matter 30, 264003 (2018).

[55] R.S. Averback and P. Bellon, in Materials Science with Ion Beams, edited by H. Bernas (Springer, Berlin, 2010), Chap. 1.

[56] M. Ghaly and R. S. Averback, Phys. Rev. Lett. 72, 364 (1994).

[57] T. Michely and C. Teichert, Phys. Rev. B 50, 11156 (1994).

[58] S. Dhar, P. Schaaf, N. Bibic, E. Hooker, M. Milosavljevic, and K. P. Lieb, Appl. Phys. A 76, 773 (2003).

[59] J. Alvarez, J. J. Hinarejos, E. G. Michel, G. R. Castro, and R. Miranda, Phys. Rev. B 45, 14042 (1992).

[60] E. G. Michel, Appl. Surf. Sci. 117/118, 294 (1997); Surface and Interface Science, edited by K. Wandelt (Wiley-VCH, Weinheim, 2014), Vol. 3, Chap. 14. 